\title{
Mechanism design without monotone differences: an example featuring buyer habits
}

\author{
Levent Ülkü
}

Received: 8 December 2013 / Accepted: 31 March 2014 / Published online: 24 April 2014 (C) SAET 2014

\begin{abstract}
Within a simple parametric example, I show that if the buyer's values fail the monotone differences condition, nonmonotone mechanisms are not just feasible but may even be desirable for a seller. The failure of monotone differences is caused by intertemporal consumption externalities in the form of habits. I show that for an interval of habit parameters the revenue maximizing mechanism is nonmonotone: the seller screens out low and high types.
\end{abstract}

Keywords Implementation $\cdot$ Monotonicity $\cdot$ Monotone differences $\cdot$ Habits

JEL Classification D42 - D61 - D82

\section{Introduction}

Consider a seller interacting with a privately informed buyer whose values exhibit an intertemporal allocation externality in the form of habit persistence: current consumption generates disutility in the future. The existence of habit renders the agent's value nonmonotone in type. I show that this nonmonotonicity leads, for a range of habit parameter values, to the optimality of a nonmonotone mechanism: as part of a revenue maximizing mechanism, the seller screens out low as well as high types, serving only to an interval of types inbetween.

L. Ülkü (凶)

Department of Economics and CIE, ITAM, Av. Santa Teresa 930, Colonia Héroes de Padierna, Delegación Magdalena Contreras, 10700 Mexico, D.F., Mexico

e-mail: levent.ulku@itam.mx 
The distinguishing feature of the environment in this paper is that the monotone differences condition need not hold. This condition leads to the possibility of ordering outcomes from "small" to "large" in such a way that larger outcomes are valued more at the margin by larger types. As a result, implementability is characterized by monotonicity of allocation functions: larger types should receive larger outcomes. In the absence of monotone differences, however, monotone allocation functions need not be implementable and implementable allocation functions need not be monotone. Importantly an optimal mechanism may involve nonmonotonicity and this is exactly what I find in a simple parametrized example, where the monotone difference condition fails for a set of parameter values.

I begin, in Sect. 2, by discussing two important background results on implementability from the literature. The first concerns a sufficient condition for implementability: if allocations can be ordered in such a way that an allocation function is monotone and the agent's values satisfy monotone differences, then the allocation function at hand is implementable. The second result is a characterization of implementability adapted from Carbajal and Ely (2013, Theorem 1) which is obtained by "integrating out" incentive constraints via the envelope theorem. This characterization also points towards a simple condition under which individual rationality follows from implementation even when payoffs are nonmonotone in types. I note this in Lemma 1 .

In Sect. 3, I analyze the example featuring a privately informed buyer with habits. For a range of habit parameters, the classical approach of Myerson (1981) can be used to construct an optimal mechanism by solving a virtual problem and checking that the solution satisfies incentive constraints. In fact this is feasible even for some (but not all) parameters which render the buyer's value nonmonotone in his type. For all sufficiently large parameters, however, the solution to the virtual problem is not implementable. In this case, I solve an optimal pricing problem which I show to be equivalent to the optimal mechanism design problem, and show that as part of an optimal mechanism the seller screens out high as well as low types. This phenomenon occurs if the habit parameter is sufficiently large, i.e., if future disutility from current consumption is sufficiently high.

There are other economic environments where the monotone differences condition fails because the agent's values are nonmonotone in his type. I construct one such environment in the concluding section, where the agent's value for an object is his expectation of an unobservable state conditional on his type, which is an informative signal. It turns out that for a class of joint densities for the state and the signal, the resulting conditional expectation is a nonmonotone function of the signal.

Berger et al. (2010) contains an example of a nonmonotone mechanism which is implementable. It has also been noted that a mechanism design problem may be solved by nonmonotone mechanisms. With single dimensional types and multidimensional outcomes, Matthews and Moore (1987) shows that a dimension of the outcome may be nonmonotone at a seller-optimum. Focusing on the class of differentiable mechanisms, García (2005) provides conditions under which the optimal mechanism is nonmonotone when the outcome space is multidimensional. The application in this paper shows that a nonmonotone optimum may exist even when both the type space and the outcome space are single dimensional. 


\section{Background results}

Consider a standard single-agent mechanism design environment. Let $A$ be a set of alternatives. An outcome is a pair $(a, p)$ where $a \in A$ and $p \in \Re$ are the agent's payment. The agent's resulting utility is $v(a, t)-p$ where $t \in T=[0,1]$ is his type. To guarantee that an envelope result applies, I will assume that $v(a, \cdot)$ is absolutely continuous and differentiable for all $a$, and I will denote its derivative at $t$ by $v_{2}(a, t)$. Throughout the text, $(s, t)$ refers to a pair of types, not necessarily distinct. Importantly, $v$ need not be linear or even monotone in $t$.

An allocation function is a function $\alpha:[0,1] \rightarrow A$ mapping types into alternatives. A mechanism is a pair $(\alpha, \pi)$ where $\alpha$ is an allocation function and $\pi:[0,1] \rightarrow \Re$ determines monetary transfers. An allocation function $\alpha$ is implementable if there exists some $\pi$ such that the agent cannot gain by misreporting his type to the mechanism $(\alpha, \pi)$, i.e., $v(\alpha(t), t)-\pi(t) \geq v(\alpha(s), t)-\pi(s)$ for every $(s, t)$. If this is the case, the mechanism $(\alpha, \pi)$ is said to be incentive compatible, or $\pi$ is said to implement $\alpha{ }^{1}$ The literature contains several related results which establish sufficient conditions for implementation of allocation functions. A typical such result indicates ${ }^{2}$ :

Proposition 1 An allocation function $\alpha$ is implementable if there exists a binary relation $\preceq$ on A such that (1) $s \leq t$ implies $\alpha(s) \preceq \alpha(t)$, and (2) $a^{\prime} \preceq$ a implies $v_{2}\left(a^{\prime}, t\right) \leq v_{2}(a, t)$ for every $t$.

The first condition in Proposition 1 orders $A$ in such a way that $\alpha$ is monotone. The second condition says that $v$ satisfies monotone differences with respect to this order on $A$ and the usual less-than-or-equal-to order on $[0,1] .{ }^{3}$ The sufficient conditions of Proposition 1 are not necessary for implementability and the allocation function in Proposition 3 below is an example to this effect. (See Remark 3 following Proposition 3.) This raises the question of whether there exists a sufficient and necessary condition for implementability. In a multidimensional setting Carbajal and Ely (2013, Theorem 1) and Berger et al. (2010, Corollary 2) give such conditions. The next proposition adapts their result in the current context. The integrals are Lebesgue.

Proposition 2 The following three statements are equivalent: (1) $\alpha$ satisfies the implementability condition

$$
\int_{s}^{t} v_{2}(\alpha(y), y) \mathrm{d} y \leq v(\alpha(t), t)-v(\alpha(t), s) \text { for every }(s, t) .
$$

\footnotetext{
1 The focus here is on incentive compatibility. If an allocation function is implementable, then it is part of an incentive compatible and individually rational mechanism under a rather mild condition. See Lemma 1 below.

2 The proofs of the results of this section are in the Appendix.

3 Proposition 1 has many precedents in various related environments. Lemma 2 of Cremer and McLean (1985) and Proposition 1 in Rochet (1987) present implementation results for arbitrary allocation functions when $A$ is an interval and comes readily ordered by the less-than-or-equal-to relation and $v$ is continuous in $a$. Proposition 3 in Bergemann and Välimäki (2002) and Theorem 5.1 in Jehiel and Moldovanu (2001) focus on efficient allocations in more abstract social choice problems, where an order on $A$ needs to be constructed in order to attain monotonicity and monotone differences.
} 
(2) The mechanism $\left(\alpha, \pi^{\alpha}\right)$ is incentive compatible where

$$
\pi^{\alpha}(t) \equiv v(\alpha(t), t)-\int_{0}^{t} v_{2}(\alpha(y), y) \mathrm{d} y \text { for every } t .
$$

(3) $\alpha$ is implementable.

In what follows, I will use the implementability of an allocation function $\alpha$ and the incentive compatibility of the mechanism $\left(\alpha, \pi^{\alpha}\right)$ as defined in (2) above interchangeably. To economize on space, I will also use the following expressions. For any allocation function $\alpha$, and any pair $(s, t)$ of types, $L_{\alpha}(s, t):=\int_{s}^{t} v_{2}(\alpha(y), y) \mathrm{d} y$ and $R_{\alpha}(s, t):=v(\alpha(t), t)-v(\alpha(t), s)$. Now the implementability condition (1) can be written as $L_{\alpha}(t, s) \geq-R_{\alpha}(s, t)$ for all $(s, t)$.

I will finish this section with a brief remark on individual rationality.

Individual rationality An important consideration in mechanism design is participation, i.e., the issue of whether truth-telling gives the agent a payoff at least as large as his outside option. It is standard to take the outside option to be zero independent of the agent's type. Suppose this is the case. I will say that $\alpha$ is individually rationally implementable if there exists some $\pi$ such that for every $(s, t)$, $v(\alpha(t), t)-\pi(t) \geq \max \{0, v(\alpha(s), t)-\pi(s)\}$. In general an implementable $\alpha$ is not necessarily individually rationally implementable. However the following lemma presents a simple condition under which individual rationality follows from incentive compatibility.

Lemma 1 An implementable $\alpha$ is individually rationally implementable if $v(\alpha(0), 0)$ $\leq v(\alpha(0), t)$ for all $t$.

If $v$ is nondecreasing in $t$, the sufficient condition in Lemma 1 trivially follows. In general, an incentive compatible mechanism is also individually rational if all types of the agent receive the same payoff from the allocation chosen for the smallest type, i.e., if $v(\alpha(0), 0)=v(\alpha(0), t)$ for all $t$.

\section{Habit environment}

In this section, I will find the optimal mechanism for a seller in a special case of the framework introduced in the previous section where

$$
\begin{aligned}
A & =\{0,1\}, \\
T & =[0,1], \text { and } \\
v(a, t) & =a t-\beta a t^{2} .
\end{aligned}
$$

The number $\beta \in(0,1)$ is a common knowledge parameter. The interpretation is as follows. The agent is purchasing an indivisible object from a seller. The allocation $a=1$ corresponds to sale and $a=0$ indicates no sale. The payment takes place at time zero. Consumption takes place at time 1 and it generates utilities in two periods: $a$ 
at time 1 and $-\beta a$ at time 2. The agent's type $t$ is his discount rate and his value $v(a, t)$ is the discounted sum of utilities. Thus as $\beta \neq 0$, the problem exhibits intertemporal allocation externalities in the form of habit formation: consumption at time 1 generates a disutility of $-\beta$ at time 2 . I will refer to $\beta$ as the habit parameter.

Note that $v_{2}(0, t)=0$ and $v_{2}(1, t)=1-2 \beta t$. Hence if $\beta>\frac{1}{2}$, these derivatives cannot be ordered independently of $t$ and Proposition 1 cannot be used to determine the implementability of any nonconstant allocation rule.

The problem of optimal mechanism design is that of finding an incentive compatible and individually rational mechanism $(\alpha, \pi)$ such that the expectation of $\pi$ is at a maximum. The formulation and solution of this problem require a statistical distribution assumption on $t$. To keep matters as simple as possible I will maintain the following assumption.

Assumption Type $t$ is uniform on [0,1].

Suppose that the seller has no costs and the buyer's outside option is zero regardless of his type. The optimal mechanism design problem is:

$$
\begin{aligned}
& \max _{(\alpha, \pi)} \int_{0}^{1} \pi(y) \mathrm{d} y \\
& \text { subject to } \begin{cases}\alpha(s)\left(s-\beta s^{2}\right)-\pi(s) \geq \alpha(t)\left(s-\beta s^{2}\right)-\pi(t) & \text { for all }(s, t), \\
\alpha(s)\left(s-\beta s^{2}\right)-\pi(s) \geq 0 & \text { for all } s .\end{cases}
\end{aligned}
$$

The next proposition identifies a solution to this problem, i.e., an optimal mechanism, for every $\beta \in(0,1)$.

Proposition 3 For every $\beta \in(0,1)$ there is an optimal mechanism $\left(\alpha^{*}, \pi^{\alpha^{*}}\right)$ given by

$$
\alpha^{*}(t)=1 \text { if and only if } \underline{\tau}(\beta) \leq t \leq \bar{\tau}(\beta)
$$

where

$$
[\underline{\tau}(\beta), \bar{\tau}(\beta)]= \begin{cases}{\left[\frac{1+\beta-\sqrt{\beta^{2}-\beta+1}}{3 \beta}, 1\right]} & \text { if } 0<\beta \leq \frac{5+\sqrt{5}}{10} \\ {\left[\frac{1-\beta}{\beta}, 1\right]} & \text { if } \frac{5+\sqrt{5}}{10}<\beta \leq \frac{3+\sqrt{3}}{6} \\ {\left[\frac{3-\sqrt{3}}{6 \beta}, \frac{3+\sqrt{3}}{6 \beta}\right]} & \text { if } \frac{3+\sqrt{3}}{6}<\beta<1\end{cases}
$$

and $\pi^{\alpha^{*}}$ is defined from $\alpha^{*}$ as in (2).

The following picture describes the optimal mechanisms. For every habit parameter $\beta$, the corresponding vertical slice of the shaded area gives the set of types that receive the object. In other words a pair $(\beta, t)$ is outside the shaded region if the optimal mechanism does not allocate the object to type $t$ when the habit parameter is $\beta$. Note that if $\beta>(3+\sqrt{3}) / 6$, then the optimal mechanism is nonmonotone: low and high types are screened out. 


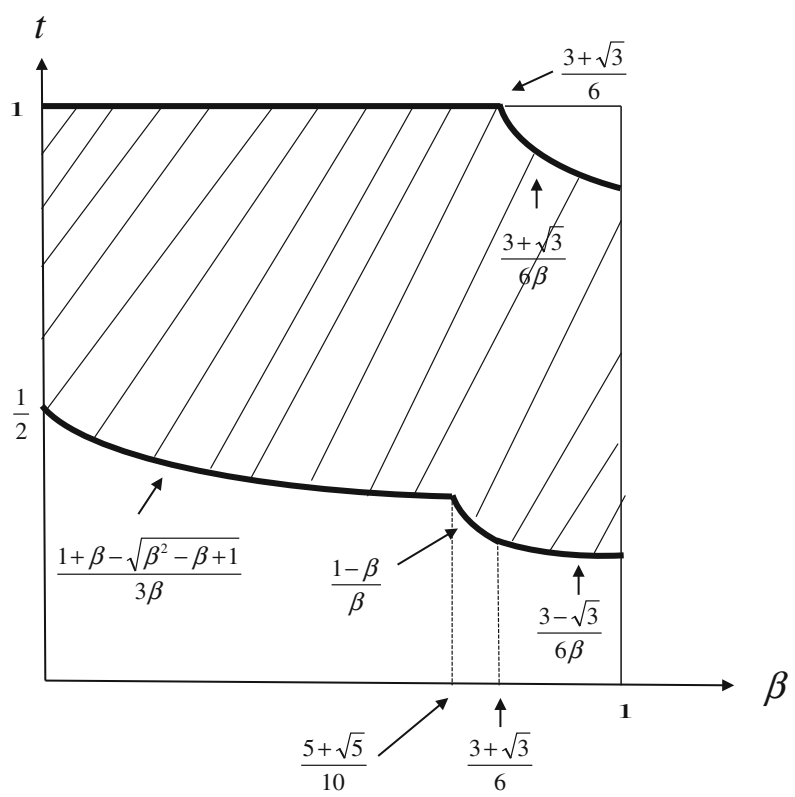

Fig. 1 Optimal mechanisms in Proposition 3

Remark 1 Since the lowest type $t=0$ is screened out for every habit parameter value, i.e., $\alpha^{*}(0)=0$ for every $\beta$, it follows that $v\left(\alpha^{*}(0), t\right)=0$ for all $t$. Now by Lemma $1,\left(\alpha^{*}, \pi^{\alpha^{*}}\right)$ is individually rational despite the nonmonotonicity of $v$ in $t$.

Remark 2 Using L'Hospital's rule $\lim _{\beta \rightarrow 0} \underline{\tau}(\beta)=\frac{1}{2}$, which is precisely the optimal cutoff type that optimally receives the object in the model with no habit formation under the uniform distribution assumption (Fig. 1).

Remark 3 Myerson (1981) provides a recipe for solving the optimal mechanism design problem which comprises of three steps. First one writes down the virtual valuations using the formula $u(a, t)=v(a, t)-\frac{1-F(t)}{F^{\prime}(t)} v_{2}(a, t)$ where $F$ is the distribution of $t$. Next one obtains an allocation function, say $\alpha^{u}$, by pointwise maximizing $u(a, t)$ at every $t$. In other words $\alpha^{u}(t) \in \arg \max _{a \in A} u(a, t)$ for all $t$. Third, one determines whether or not $\alpha^{u}$ is individually rationally implementable. If so, then it is part of an optimal mechanism, with payments given as in (2). If the agent's value is given by $v(a, t)=a t$, then this follows under the increasing hazard rate assumption on $F$. In my problem, under the uniformity assumption,

$$
\begin{aligned}
u(a, t) & =v(a, t)-(1-t) v_{2}(a, t) \\
& =a\left[\left(t-\beta t^{2}\right)-(1-t)(1-2 \beta t)\right] \\
& =a\left[-3 \beta t^{2}+2(1+\beta) t-1\right],
\end{aligned}
$$


and the solution to the pointwise "virtual problem" $\max _{a \in\{0,1\}} u(a, t)$ yields the allocation function

$$
\alpha^{u}(t)= \begin{cases}1 & \text { if } \frac{1+\beta-\sqrt{\beta^{2}-\beta+1}}{3 \beta} \leq t \leq 1, \text { and } \\ 0 & \text { if } 0 \leq t<\frac{1+\beta-\sqrt{\beta^{2}-\beta+1}}{3 \beta} .\end{cases}
$$

Exactly as in Myerson (1981), if $\alpha^{u}$ is individually rationally implementable, then it is part of an optimal mechanism in my problem. Note that $\alpha^{u}(0)=0$, therefore individual rationality will follow from implementability by Lemma 1 . Since I have already made an assumption on the distribution of types, the implementability of $\alpha^{u}$, in return, solely depends on the value of the habit parameter $\beta$.

Case 1 Suppose that $\beta \in\left(0, \frac{1}{2}\right]$ so that values satisfy monotone differences when $A$ is ordered by $0 \preceq 1: v_{2}(0, t)=0 \leq 1-2 \beta=v_{2}(1, t)$ for all $t$. Now Proposition 1 indicates that all monotone allocation functions are implementable. Consequently, since it is monotone, $\alpha^{v}$ is implementable and therefore part of the optimal mechanism.

Case 2 If $\beta \in\left(\frac{1}{2}, \frac{5+\sqrt{5}}{10}\right]$, then Proposition 1 cannot be used to determine the implementability of the non-constant allocation function $\alpha^{v}$. Yet, the proof of Proposition 3 below shows that $\alpha^{v}$ nevertheless satisfies the implementability condition (1). Hence Myerson's recipe can be used to solve for a monotone optimal mechanism even in the absence of monotone differences.

Case 3 If $\beta \in\left(\frac{5+\sqrt{5}}{10}, 1\right]$, on the other hand, $\alpha^{v}$ fails the implementability condition (1). To see this note that

$$
L_{\alpha^{v}}(1,0)=\underline{\tau}(\beta)-\beta \underline{\tau}^{2}(\beta)-1+\beta>0=R_{\alpha^{v}}(1,0),
$$

in other words, type $s=1$ has a strict incentive to report $t=0$ to the mecha$\operatorname{nism}\left(\alpha^{v}, \pi^{\alpha^{v}}\right)$.

Remark 4 The optimal mechanism for Case 3 can be solved for using the observation that every implementable allocation can be identified with some take-it-or-leave-it offer at a fixed price and that solving for the optimal take-it-or-leave-it price yields indirectly the optimal mechanism. I will show in particular that if $\beta$ is high enough, namely if $\frac{3+\sqrt{3}}{6}<\beta$, then the optimal mechanism obtained this way is nonmonotone: low and high values of $t$ are screened out by the seller. This is intuitive: high types discount future habit cost at a higher rate, leading to a lower overall value for the allocation. If this cost is large enough, then the seller is better off excluding high types in favor of types with higher discounted values.

I complete with the proof of Proposition 3.

Proof of Proposition 3 The proof is divided into three parts depending on the value of the habit parameter $\beta$. In the first two parts where $\beta \in(0,1 / 2]$ and $\beta \in$ $(1 / 2,(5+\sqrt{5}) / 10]$, respectively, the allocation function $\alpha^{*}$ defined in the result and the solution to the virtual problem $\alpha^{v}$ given in (3) are identical. Using the methods of Myerson (1981), then, the mechanism $\left(\alpha^{*}, \pi^{\alpha^{*}}\right)$ is optimal if $\alpha^{*}$ is implementable 
and $\left(\alpha^{*}, \pi^{\alpha^{*}}\right)$ is individually rational. Individual rationality follows from Lemma 1 and thus the proof boils down to showing that $\alpha^{*}$ is implementable in these cases. In part 1 , I do so using Proposition 1 and monotone differences. In part 2, I use the characterization in Proposition 2.

In Part 3, when $\beta$ is sufficiently high, $\alpha^{*} \neq \alpha^{v}$. In this case I establish the optimality of $\alpha^{*}$ by solving an optimal pricing problem which is equivalent to the optimal mechanism design problem. I show that $\alpha^{*}$ is the allocation supported by the optimal price, and for any type $t$ such that $\alpha^{*}(t)=1, \pi^{\alpha^{*}}(t)$ is the optimal price.

Part 1 Suppose that $\beta \in(0,1 / 2]$. Since $\alpha^{*}=\alpha^{v}$, I need only show that $\left(\alpha^{*}, \pi^{\alpha^{*}}\right)$ is feasible in the optimal mechanism design problem. The monotone difference property is satisfied when the allocations are ordered $0 \preceq 1$, as $v_{2}(0, t)=0 \leq 1-2 \beta t=$ $v_{2}(1, t)$ for all $t$. Now $\alpha^{*}$ is nondecreasing and therefore implementable by Proposition 1. Furthermore, since $\alpha^{*}(0)=0$ and $v(0, t)=0$ for all $t$, Lemma 1 applies and $\left(\alpha^{*}, \pi^{\alpha^{*}}\right)$ is also individually rational.

Part 2 Suppose that $\beta \in(1 / 2,(5+\sqrt{5}) / 10]$. As in part $1, \alpha^{*}=\alpha^{v}$ and I need to only show that $\left(\alpha^{*}, \pi^{\alpha^{*}}\right)$ is incentive compatible and individually rational. Again, as in part 1, Lemma 1 implies that individual rationality follows from incentive compatibility since $\alpha^{*}(0)=0$. Hence I need to only show that $\left(\alpha^{*}, \pi^{\alpha^{*}}\right)$ is incentive compatible, or $\alpha^{*}$ satisfies (1) in Proposition 2.

To begin, note that the following numbers are ordered:

$$
0<\underline{\tau}(\beta)<1 / 2 \beta<1<1 / \beta \text {. }
$$

Take any $(s, t)$ such that $s<\underline{\tau}(\beta) \leq t$. It follows that

$$
\begin{aligned}
L_{\alpha^{*}}(s, t) & =\int_{\underline{\tau}(\beta)}^{t}(1-2 \beta y) \mathrm{d} y \\
& =t-\underline{\tau}(\beta)-\beta t^{2}+\beta[\underline{\tau}(\beta)]^{2} \\
& <t-s-\beta t^{2}+\beta s^{2} \\
& =R_{\alpha^{*}}(s, t)
\end{aligned}
$$

where the inequality follows because $s<\underline{\tau}(\beta)<1 / 2 \beta$.

Now take $(s, t)$ such that $t<\tau(\beta) \leq s$ so that

$$
\begin{aligned}
L_{\alpha^{*}}(s, t) & =\int_{s}^{\underline{\tau}(\beta)}(1-2 \beta y) \mathrm{d} y \\
& =\underline{\tau}(\beta)-s-\beta[\underline{\tau}(\beta)]^{2}+\beta s^{2} \\
& \leq 0 \\
& =R_{\alpha^{*}}(s, t) .
\end{aligned}
$$

The weak inequality follows from the following observations: let $f(y)=y-\beta y^{2}$ on $[0,1]$. I need to show $f(\underline{\tau}(\beta)) \leq f(s)$. Note that $f$ is concave and maximized at 
$1 / 2 \beta$. Hence if $\underline{\tau}(\beta) \leq s \leq 1 / 2 \beta$, the inequality follows trivially. Otherwise, note that

$$
\min _{y \in(1 / 2 \beta, 1]} f(y)=f(1)=1-\beta .
$$

Thus, it suffices to show that $f(\underline{\tau}(\beta)) \leq f(1)$. But this follows as $\beta \leq(5+\sqrt{5}) / 10$. Thus $\alpha^{*}$ satisfies (1) and is therefore implementable.

Part 3 If $\beta>(5+\sqrt{5}) / 10$, then $\alpha^{v}$ is not implementable as I argued in Remark 3 above. Hence Myerson's methods cannot be used to solve for an optimal mechanism. I will instead derive the optimal mechanism by solving an optimal pricing problem. I need some preparations. Let $p \geq 0$ be a price. If the seller makes a take-it-or-leave-it offer at $p$, then any type whose value weakly exceeds $p$ will purchase the object. Hence each $p$ gives rise to an allocation function $\alpha^{p}$ defined by

$$
\alpha^{p}(t)= \begin{cases}1 & \text { if } t-\beta t^{2} \geq p \\ 0 & \text { otherwise }\end{cases}
$$

Step 1 Rewrite $\alpha^{p}$ as follows:

$$
\alpha^{p}(t)= \begin{cases}1 & \text { if } \frac{1-\sqrt{1-4 \beta p}}{2 \beta} \leq t \leq \frac{1+\sqrt{1-4 \beta p}}{2 \beta} \\ 0 & \text { otherwise. }\end{cases}
$$

Calculating the payments $\pi^{\alpha^{p}}$ as in (2), I get

$$
\pi^{\alpha^{p}}(t)= \begin{cases}p & \text { if } \frac{1-\sqrt{1-4 \beta p}}{2 \beta} \leq t \leq \frac{1+\sqrt{1-4 \beta p}}{2 \beta} \\ 0 & \text { otherwise }\end{cases}
$$

Hence if a type $t$ receives the object, she makes the payment $p$. Now one could directly check that the direct mechanism $\left(\alpha^{p}, \pi^{\alpha^{p}}\right)$ is incentive compatible. This is tantamount to saying that the agent's best response to the take-it-or-leave-it offer at $p$ is to purchase the object if $t-\beta t^{2} \geq p$, and to pass otherwise. I skip this straightforward step. ${ }^{4}$

Step 2 On the other hand, if a mechanism $\left(\alpha, \pi^{\alpha}\right)$ is implementable, then it satisfies the following condition:

$$
\text { if } \alpha(s)=1 \text { and } t-\beta t^{2}>s-\beta s^{2} \quad \text { then } \alpha(t)=1 .
$$

If not, then for some $(s, t)$ such that $\alpha(s)=1, \alpha(t)=0$ and $t-\beta t^{2}>s-\beta s^{2}$, we would have

$$
L_{\alpha}(s, t) \leq R_{\alpha}(s, t)=0
$$

\footnotetext{
4 Alternatively, using Proposition 2, one could check that the allocation function $\alpha^{p}$ satisfies the implementability condition (1) directly.
} 
and

$$
0 \leq-L_{\alpha}(s, t)=L_{\alpha}(t, s) \leq R_{\alpha}(t, s)=s-\beta s^{2}-t+\beta t^{2}<0,
$$

a contradiction to implementability of $\alpha$. Condition (*) in turn implies that the allocation function $\alpha$ is supported by a take-it-or-leave-it price at $p=t_{0}+\beta t_{0}^{2}$ where $t_{0}=\inf \{t: \alpha(t)=1\}$. But $t_{0}+\beta t_{0}^{2}$ is precisely $\pi^{\alpha}(t)$ for any $t$ such that $\alpha(t)=1$. In other words, any incentive compatible mechanism $\left(\alpha, \pi^{\alpha}\right)$ can be identified by a take-it-or-leave-it offer.

Step 3 To solve for the optimal mechanism, then, I need only solve for the optimal price $p$ for the seller and then convert it to the equivalent direct mechanism. To formulate the optimal pricing problem, first fix any habit parameter $\beta \in\left(\frac{\sqrt{5}+5}{10}, 1\right)$. Using the uniformity of $t$, for every price $p \geq 0$, write the expected revenue of the seller as

$$
\pi(p \mid \beta)=p \operatorname{Pr}\left\{t-\beta t^{2} \geq p\right\}= \begin{cases}p\left(1-\frac{1-\sqrt{1-4 \beta p}}{2 \beta}\right) & \text { if } 0 \leq p \leq 1-\beta, \\ \frac{p \sqrt{1-4 \beta p}}{\beta} & \text { if } 1-\beta<p \leq \frac{1}{4 \beta} \\ 0 & \text { if } \frac{1}{4 \beta}<p .\end{cases}
$$

Note that $0<1-\beta<\frac{1}{4 \beta}$ and therefore the expected revenue function is well-defined. Also note that $\pi(\cdot \mid \beta)$ is continuous. To show this I need only check continuity at the kinks which occur at $p=1-\beta$ and $p=\frac{1}{4 \beta}$. Continuity from the left is obvious. Note for any $\beta$

$$
\begin{aligned}
\lim _{p \downarrow 1-\beta} \pi(p \mid \beta) & =\lim _{p \downarrow 1-\beta} \frac{p \sqrt{1-4 \beta p}}{\beta} \\
& =\frac{(1-\beta) \sqrt{1-4 \beta(1-\beta)}}{\beta} \\
& =\frac{(1-\beta)(2 \beta-1)}{\beta} \\
& =\pi(1-\beta \mid \beta)
\end{aligned}
$$

and continuity at $1-\beta$ follows. Furthermore $\pi(p \mid \beta)$ is also continuous at $p=\frac{1}{4 \beta}$ since $\pi\left(\frac{1}{4 \beta} \mid \beta\right)=0=\pi(p \mid \beta)$ for all $p>\frac{1}{4 \beta}$.

Step 4 Now I will solve the optimal pricing problem

$$
\max _{p} \pi(p \mid \beta)
$$

piece-by-piece, first on the domain $[0,1-\beta]$, and then on $\left(1-\beta, \frac{1}{4 \beta}\right]$.

I claim that

$$
\{1-\beta\}=\arg \max _{0 \leq p \leq 1-\beta} \pi(p \mid \beta) .
$$


To see this, first note that $\pi(0 \mid \beta)=0$. Moreover for every $p \in[0,1-\beta]$, the first and second derivatives of expected revenue with respect to price are

$$
\begin{aligned}
& \pi^{\prime}(p \mid \beta)=1+\frac{1}{2 \beta}(\sqrt{1-4 p \beta}-1)-\frac{p}{\sqrt{1-4 p \beta}} \\
& \pi^{\prime \prime}(p \mid \beta)=-2 p \frac{\beta}{(1-4 p \beta)^{\frac{3}{2}}}-\frac{2}{\sqrt{1-4 p \beta}}=-2<0
\end{aligned}
$$

Thus $\pi(\cdot \mid \beta)$ is strictly concave on $[0,1-\beta]$. The first derivative evaluated at $p=0$ is $\pi^{\prime}(0 \mid \beta)=1>0$, and therefore the expected revenue is increasing around 0 . Finally the first derivative evaluated at $p=1-\beta$ is

$$
\pi^{\prime}(1-\beta \mid \beta)=\frac{\beta-1}{\beta}-\frac{1-\beta}{2 \beta-1}+1>0 .
$$

[In fact $\pi^{\prime}(1-\beta \mid \beta)=0$ if $\beta=\frac{\sqrt{5}+5}{10}$.] Thus $\pi(\cdot \mid \beta)$ is strictly increasing on $[0,1-\beta]$ and is maximized at $p=1-\beta$.

Next, I note that

$$
\begin{array}{r}
\beta \in\left(\frac{\sqrt{3}+3}{6}, 1\right) \Rightarrow \arg \max _{1-\beta<p \leq \frac{1}{4 \beta}} \pi(p \mid \beta)=\left\{\frac{1}{6 \beta}\right\}, \text { and } \\
\beta \in\left(\frac{\sqrt{5}+5}{10}, \frac{\sqrt{3}+3}{6}\right] \Rightarrow \pi(1-\beta \mid \beta)=\sup _{1-\beta<p \leq \frac{1}{4 \beta}} \pi(p \mid \beta) .
\end{array}
$$

Consequently the expected revenue maximizing price is

$$
p^{*}(\beta)= \begin{cases}\frac{1}{6 \beta} & \text { if } \frac{\sqrt{3}+3}{6}<\beta<1, \text { and } \\ 1-\beta & \text { if } \frac{\sqrt{5}+5}{10}<\beta \leq \frac{\sqrt{3}+3}{6}\end{cases}
$$

All that remains to show is that the allocation function $\alpha^{*}$ in Proposition 3 is such that for every $\beta \geq \frac{5+\sqrt{5}}{10}, \alpha^{*}(t)=1$ if and only if $t-\beta t^{2} \geq p^{*}(\beta)$ using the definition in the ultimate display. I skip this straightforward step and the proof is complete.

\section{Conclusion}

In this paper I solve for seller-optimal mechanisms in an environment where the buyer has habits. The critical feature of the environment is that the buyer's values fail the monotone differences property. I show that the optimal mechanism could, for a range of habit parameters, be nonmonotone in the agent's type.

I conclude by describing a different economic environment where the buyer's value is nonmonotone in his type and the monotone differences condition fails for any nontrivial ordering of outcomes. Suppose that an agent's value for an object is some 
unobservable state $\theta$ which takes values in $[0,1]$. The type $t \in[0,1]$ is an informative signal regarding $\theta$. Consider the following joint density for $(\theta, t)$ :

$$
g(\theta, t)= \begin{cases}2 & \text { if }(\theta, t) \in[0,1]^{2} \text { and } \theta \geq \max \{1-2 t, 2 t-1\}, \\ 0 & \text { otherwise. }\end{cases}
$$

The signal technology can be interpreted as follows. The conditional density $g(t \mid \theta)$ is uniform on $\left[\frac{1-\theta}{2}, \frac{1+\theta}{2}\right]$. Thus higher values of the state induce less precise signals while keeping the conditional expectation of the signal always the same at $\frac{1}{2}$. Note that the conditional density $g(\theta \mid t)$ is uniform on $[1-2 t, 1]$ if $t \in\left[0, \frac{1}{2}\right]$ and it is uniform on $[2 t-1,1]$ if $t \in\left[\frac{1}{2}, 1\right]$. Consequently the agent's conditional expectation of state $\theta$ given his signal $t$ is

$$
E[\theta \mid t]= \begin{cases}1-t & \text { if } t \in\left[0, \frac{1}{2}\right] \\ t & \text { if } t \in\left[\frac{1}{2}, 1\right]\end{cases}
$$

Now set $v(a, t)=a E[\theta \mid t]$ for $a \in\{0,1\}$ so that $v(1, t)$ is nonmonotone and $v(0, t)=$ 0 . Despite the failure of differentiability at $\frac{1}{2}, v$ is convex in $t$ and an appropriate envelope result will imply that condition (1) characterizes implementability. It can be shown that implementable allocations are typically nonmonotone in this environment. Furthermore one can solve for the optimal mechanism using Proposition 2 and Lemma 1 in an argument similar to the last part of the proof of Proposition 3, i.e., by solving an optimal pricing problem.

\section{Appendix: Proofs of the results in Sect. 2}

Proof of Proposition 1 Using Proposition 2-whose proof is to follow-I need only show that if there is an order $\preceq$ on $A$ such that (i) $s \leq t$ implies $\alpha(s) \preceq \alpha(t)$, and (ii) $a^{\prime} \preceq a$ implies $v_{2}\left(a^{\prime}, t\right) \leq v_{2}(a, t)$ for every $t$, then the implementability condition (1) follows. Suppose such $\preceq$ exists and take $s<t$. Then

$$
\int_{s}^{t} v_{2}(\alpha(y), y) \mathrm{d} y \leq \int_{s}^{t} v_{2}(\alpha(t), y) \mathrm{d} y=v(\alpha(t), t)-v(\alpha(t), s) .
$$

Similarly if $t<s$. Thus $\alpha$ satisfies (1) and the proof is complete.

Proof of Proposition $2(1 \Rightarrow 2)$ Suppose that $\alpha$ satisfies (1). Then the mechanism $\left(\alpha, \pi^{\alpha}\right)$ is incentive compatible as for every $(s, t)$

$\pi^{\alpha}(t)-\pi^{\alpha}(s)=v(\alpha(t), t)-v(\alpha(s), s)-\int_{s}^{t} v_{2}(\alpha(y), y) \mathrm{d} y \geq v(\alpha(t), s)-v(\alpha(s), s)$.

$(2 \Rightarrow 3)$ This trivially follows from definitions. 
$(3 \Rightarrow 1)$ To see that $(1)$ is necessary for implementation, suppose that for some payment function $\pi,(\alpha, \pi)$ is incentive compatible. Since $v$ is absolutely continuous and differentiable in types, the envelope theorem of Milgrom and Segal (2002) applies and

$$
v(\alpha(t), t)-\pi(t)=v(\alpha(s), s)-\pi(s)+\int_{s}^{t} v_{2}(\alpha(y), y) \mathrm{d} y
$$

for every $(s, t)$. Rearranging and using incentive compatibility,

$$
\begin{aligned}
v(\alpha(t), t) & =v(\alpha(s), s)-\pi(s)+\pi(t)+\int_{s}^{t} v_{2}(\alpha(y), y) \mathrm{d} y \\
& \geq v(\alpha(t), s)+\int_{s}^{t} v_{2}(\alpha(y), y) \mathrm{d} y
\end{aligned}
$$

and (1) follows.

Proof of Lemma 1 Suppose that $\alpha$ is implementable and $v(\alpha(0), t) \geq v(\alpha(0), 0)$ for all $t$. Since $\left(\alpha, \pi^{\alpha}\right)$ is incentive compatible (Proposition 2), we have $v(\alpha(t), t)-$ $\pi^{\alpha}(t)=L_{\alpha}(0, t) \geq-R_{\alpha}(t, 0) \geq 0$, where the equality follows from the definition of payments $\pi^{\alpha}$ in (2), the first inequality follows from the implementation condition (1), and the second inequality follows from hypothesis.

\section{References}

Bergemann, D., Välimäki, J.: Information acquisition and efficient mechanism design. Econometrica 70, 1007-1034 (2002)

Berger, A., Müller, R., Naaemi, S.H.: Path-Monotonicity and Incentive Compatibility. METEOR Research Memorandum, Maastricht University, Maastricht (2010)

Branco, F.: Multiple unit auctions of an indivisible good. Econ. Theory 8, 77-101 (1996)

Carbajal, J.C., Ely, J.: Mechanism design without revenue equivalence. J. Econ. Theory 148(1), 104-133 (2013)

Cremer, J., McLean, R.P.: Optimal selling strategies for a discriminating monopolist when demands are interdependent. Econometrica 53, 345-361 (1985)

García, D.: Monotonicity in direct revelation mechanisms. Econ. Lett. 88(1), 21-26 (2005)

Jehiel, P., Moldovanu, B.: Efficient design with interdependent valuations. Econometrica 69, 1237-1259 (2001)

Matthews, S., Moore, J.: Monopoly provision of quality and warranties: an exploration in the theory of multidimensional screening. Econometrica 55(2), 441-467 (1987)

Milgrom, P., Segal, I.: Envelope theorems for arbitrary choice sets. Econometrica 70, 583-601 (2002)

Milgrom, P., Shannon, C.: Monotone comparative statics. Econometrica 62, 157-180 (1994)

Mookherjee, D., Reichelstein, S.: Dominant strategy implementation of Bayesian incentive compatible allocation rules. J. Econ. Theory 56(2), 378-399 (1992)

Myerson, R.: Optimal auction design. Math. Oper. Res. 6, 58-73 (1981)

Rochet, J.C.: A necessary and sufficient condition for rationalizability in a quasi-linear context. J. Math. Econ. 16(2), 191-200 (1987)

Ülkü, L.: Optimal combinatorial mechanism design. Econ. Theory 53(2), 473-498 (2013) 